University of Wollongong

Research Online

Faculty of Education - Papers (Archive)

Faculty of Arts, Social Sciences \& Humanities

$1-1-2017$

\title{
Muscular fitness, adherence to the Southern European Atlantic Diet and cardiometabolic risk factors in adolescents
}

Cesar A. Agostinis-Sobrinho

University of Porto

Sandra Abreu

University of Porto

Carla Moreira

University of Porto

Luis Lopes

University of Porto

Antonio García-Hermoso

University of Santiago

See next page for additional authors

Follow this and additional works at: https://ro.uow.edu.au/edupapers

Part of the Education Commons

\section{Recommended Citation}

Agostinis-Sobrinho, Cesar A.; Abreu, Sandra; Moreira, Carla; Lopes, Luis; García-Hermoso, Antonio; Ramírez-Velez, R; Correa-Bautista, Jorge Enrique; Mota, Jorge; and Santos, Rute: Muscular fitness, adherence to the Southern European Atlantic Diet and cardiometabolic risk factors in adolescents 2017, 695-702.

https://ro.uow.edu.au/edupapers/1238

Research Online is the open access institutional repository for the University of Wollongong. For further information contact the UOW Library: research-pubs@uow.edu.au 


\section{Authors}

Cesar A. Agostinis-Sobrinho, Sandra Abreu, Carla Moreira, Luis Lopes, Antonio García-Hermoso, R Ramírez-Velez, Jorge Enrique Correa-Bautista, Jorge Mota, and Rute Santos 
1 Muscular Fitness, adherence to the Southern European Atlantic Diet and cardiometabolic risk factors in adolescents.

3 Agostinis-Sobrinho, César ${ }^{1}$; Abreu, Sandra.; Moreira, Carla ${ }^{1}$; Lopes, Luís ${ }^{1}$; García4 Hermoso, Antonio ${ }^{2}$; Ramírez-Vélez, Robinson ${ }^{3}$; Correa-Bautista, Jorge Enrique ${ }^{3}$; 5 Mota, Jorge $^{1} \&$ Santos, Rute ${ }^{1,4}$

\section{Corresponding Author:}

César Aparecido Agostinis Sobrinho

Research Centre in Physical Activity, Health and Leisure

Faculty of Sport - University of Porto

Rua Dr. Plácido Costa, 91. 4200-450 Porto

Phone number: 00351225074786

Fax number: 00351225500689

Email: cesaragostinis@hotmail.com

${ }^{1}$ Research Centre in Physical Activity, Health and Leisure, Faculty of Sport, University

${ }^{2}$ Physical Activity, Sport and Health Sciences Laboratory, University of Santiago de Chile, Santiago de Chile, Chile

Physical Activity Measurements, School of Medicine and Health Sciences, University of Rosario, Bogotá

University of Wollongong, Australia. 
KEYWORDS: Metabolic syndrome, Muscle strength, diet pattern, Youth.

\section{Abstract}

Background: Muscular fitness and an adherence to the Southern European Atlantic Diet (SEADiet) have been inversely associated with cardiometabolic risk.

Aim: To assess the independent and combined associations of muscular fitness and adherence to the SEADiet on cardiometabolic risk in adolescents.

Material and methods: A total of 467 Portuguese adolescents (275 girls) participated in this cross-sectional study. Sum of the Z-Scores of Curl-Up and Push-Up tests was used to create a muscular fitness score. Adherence to SEADiet was obtained using a food frequency questionnaire. A cardiometabolic risk score was computed from sum of Zscore of triglycerides, systolic blood pressure, total cholesterol/HDL ratio, HOMA-IR and waist circumference.

Results: Adolescents with low muscular fitness and low adherence to the SEADiet had the poorest cardiovascular profile $\left.\mathrm{F}_{(5,452)}=5.074 p<0.001\right)$ and the highest odds of having a high cardiometabolic risk score $(\mathrm{OR}=4.5$; 95\% $\mathrm{CI}: 2.1-14)$ when compared to those with High muscular fitness / High adherence to the SEADiet after adjustments for age, sex, pubertal stage, socioeconomic status, total energy intake, low-energy reporter and cardiorespiratory fitness..

Conclusions: Our findings seem suggest that improving muscular fitness as well as an adherence to the SEADiet could be an important strategy to reduce clustered cardiometabolic risk in youth. 

countries [1]. The precursors of cardiovascular diseases have their onset in childhood [2]. The most recognised cardiovascular risk factors in paediatric age are blood pressure, triglycerides, total cholesterol (TC), high-density lipoprotein cholesterol (HDL), insulin resistance, and waist circumference $[3,4]$.

Unhealthy lifestyles are linked to metabolic and CVD [5], inducing endothelial dysfunction, accelerating progression of atherosclerosis, and increasing mortality [5-7]. There is an unequivocal association between poor physical fitness and cardiovascular risk factors in childhood and adolescence [8]. However, recently there has also been an increased interest on the effects of muscle strength on metabolically healthy subjects [9]. In adults, muscular fitness is inversely and independently associated with all-cause mortality, even after adjusting for several potential confounders [10]. Epidemiological studies show that higher muscle strength is associated with ideal cardiovascular health in adolescents [11] and is inversely associated with cardiovascular disease and cardiometabolic risk factors $[9,12-14]$.

Dietary habits also play an important role in the development and progression of CVD. The Southern European Atlantic Diet (the SEADiet) is the traditional diet of northern Portugal and northwest Spain, characterized by high a consumption of fish, red meat, dairy products, vegetables and legumes, vegetable soup, potatoes, whole wheat bread, and wine [15]. The adherence to SEADiet has been associated with decreased myocardial infarction risk due to its association with lower concentrations of inflammatory markers, reduced triglycerides, insulin, insulin resistance, and systolic blood pressure [16].

Some studies have investigated the combined effect of cardiorespiratory fitness and dietary pattern on cardiovascular risk factors $[17,18]$. However, the combined 
95 association of diet and muscular fitness on cardiovascular risk factors among 96 adolescents is largely unknown. Nevertheless, it is known that skeletal muscle is the 97 largest organ in the human body, which expresses and releases several cytokines in 98 response to muscle contractions, acting as an endocrine organ [19]. In this line, it seems 99 important to understand the interplay between muscular fitness, diet, and cardiovascular 100 risk factors.

Therefore, the present study aimed to assess the impact of the independent and 102 combined associations of muscular fitness and adherence to the SEADiet on the clustering of cardiometabolic risk factors in adolescents. 


\section{Study Design and Sample}

107

Data for the present study derived from a school-based study, the Azorean

Physical Activity and Health Study II, aimed to evaluate physical activity (PA), physical fitness, and prevalence of overweight/obesity, dietary intake, health-related quality of life and related factors. The study was carried out in six of the nine Azorean Islands - Portugal (S. Miguel, Terceira, Faial, Pico, S. Jorge, and Graciosa) where 95\% of the population lives. Details on the study design and sampling strategy are reported elsewhere [20]. For this study, we only considered the 467 adolescents with metabolic data evaluated in 2009 and that have completed all interest variables (275 girls and 192 boys) aged 15 to 18 (mean age 16.5). All participants in this study were informed about the objectives of the work, and parents or guardians provided written informed consent. The study was approved by the Faculty of Sport at the University of Porto and the Portuguese Foundation for Science and Technology ethics committee and was conducted in accordance to the declaration of Helsinki for Human Studies of the World Medical Association.

\section{Measures}

\section{Muscular fitness}

The Curl Up and Push Up tests were used to evaluate the muscular fitness. All tests were conducted according to the Fitnessgram measurement procedures [21]. The curl-up test was used to evaluate abdominal strength and endurance. Participants lie down with knees bent and feet unanchored. Set to a specified pace, adolescents 
complete as many repetitions as possible to a maximum of 75 .

The push-up test was used to evaluate upper body strength and endurance. Participants lower the body to a 90- degree elbow angle and push up. Set to a specified pace, adolescents complete as many repetitions as possible.

The results of the Push up and Curl up tests (number of repetitions) were transformed into standardized values (Z-scores) [(participant's value e mean value of the sample)/SD] by age and sex. Then the sum of Z-Scores of the two tests was performed to create an overall muscular fitness score. Participants were divided in three groups (by tertiles of muscular fitness score, by age and sex): low, medium and high muscular fitness $[13,14]$. Thus, in according of SEADiet profile and muscular fitness level, six exclusive groups were created.

\section{Southern European Atlantic Diet score}

3

Dietary intake was obtained using a self-administered, semi-quantitative food frequency questionnaire (FFQ) regarding the previous 12 months, validated for Portuguese adults $[22,23]$ The FFQ was adapted for adolescents by including foods more frequently eaten by this age [23,24] comprising 91 food items or beverage categories [25]. Food intake was calculated by weight in one of the nine possibilities of frequency of consumption (from never or less than once per month, to six or more times a day), by the weight of the standard portion size of the food-item. Energy and nutritional intake were estimated with consideration of the respondents' ratings of frequency, portion, and seasonality of each item, using the software Food Processor Plus (ESHA Research Inc, Salem, OR, US). This program uses nutritional information 
154 from the United States that has been adapted for use with typical Portuguese foods and

155 beverages. The Cronbach's $\alpha$, to test FFQ inter-item consistency, was $(\alpha=.892)$.

The adherence to the traditional Southern European Diet was assessed by the originally constructed based on the intake of nine food groups, namely fresh fish (excluding cod), cod, red meat and pork products, dairy products, legumes and vegetables, vegetable soup, potatoes, whole-grain bread and wine. Each component (except wine) was measured as grams per $1000 \mathrm{kcal}$ per day. Using the sex-specific median of the study's participants as a cut-off value for each of the components, 1 point was given when intake $>=$ median and 0 points for intakes below median for all items except for wine ( $<=1$ glass/d in women and $<=2$ glasses $/ \mathrm{d}$ in men $=1$ point). In the present study, we adapted this score by attributing 0 points to any wine consumption, since ethanol consumption is not recommended for children and adolescents. If participants met all the characteristics of SEADiet, their score was the highest (nine points), reflecting maximum adherence. If they met none of the characteristics, the score was minimum (zero), reflecting no adherence. Based on these results, participants were categorized into two groups: low $(<=4$ points $)$ and high $(>=5$ points $)$ adherence accordingly with the sample's median.

\section{Cardiorespiratory Fitness}

Cardiorespiratory fitness (CRF) was assessed with the 20-metre Shuttle Run Test (20 m SRT) [26]. This test requires participants to run back and forth between two

177 lines set $20 \mathrm{~m}$ apart. Running speed started at $8.5 \mathrm{~km} / \mathrm{h}$ and increased by $0.5 \mathrm{~km} / \mathrm{h}$ each minute, reaching $18.0 \mathrm{~km} / \mathrm{h}$ at minute 20 . Each level was announced on a tape player. 
179 The participants were instructed to keep up with the pace until exhausted. The test was

180 finished when the participant failed to reach the end lines concurrent with the audio 181 signals on two consecutive occasions. Otherwise, the test ended when the subject 182 stopped because of fatigue. The participants received verbal encouragements from the 183 investigators to achieve maximum performance, to keep running as long as possible. 184 Number of shuttles performed by each participant was recorded.

\section{Anthropometrics}

Height was measured to the nearest millimeter $(0.1 \mathrm{~cm})$ in bare or stocking feet with the adolescent standing upright against a stadiometer (Holtain Ltd., Crymmych, Pembroke shire, UK). Weight was measured to the nearest $0.10 \mathrm{~kg}$, with adolescents lightly dressed using a portable electronic weight scale (Tanita Inner Scan BC 532). Waist Circumference (WC) measurement was taken in a standing position, to the nearest $0.1 \mathrm{~cm}$, with a tape measure midway between the lower rib margin and the anterior superior iliac spine at the end of normal expiration. WC measurements was taken as described by Lohman[27].

\section{Pubertal stage}

Participants self-assessed their pubertal stage of secondary sex characteristics (breast and pubic hair development for girls, genital and pubic hair development for boys; ranging from stage I to V), according to the criteria of Tanner and Whitehouse [28]. 


\section{Parental Education level}

The highest level of parental education (in completed years of education) was considered as a proxy of socioeconomic status. Similar procedures have also been applied in the Portuguese context [29]

\section{Resting Blood Pressure}

Blood pressure was measured using a Dynamap vital signs monitors (model BP 8800, Critikon, Inc., Tampa, Florida). Trained nurses took measurements, and all adolescents were required to sit and rest for at least $5 \mathrm{~min}$ prior to the first blood pressure measurement. Participants were in a seated, relaxed position with their feet resting flat on the ground. Two measurements in the non-dominant arm were taken, after five and $10 \mathrm{~min}$ of rest. The mean of these two measurements was used for statistical analysis. If the two measurements differed by two $\mathrm{mmHg}$ or more, a third measure was taken [30].

\section{Blood Sampling}

Blood samples were obtained from each subject early in the morning, following a 10-hour overnight fast by venipuncture from the antecubital vein. The samples were stored in sterile blood collection tubes in refrigerated conditions $\left(4^{\circ}\right.$ to $8^{\circ} \mathrm{C}$ ) for no longer than 4 hours during the morning of collection and then sent to an analytical laboratory for testing according to standardized procedures, as follow: Serum glucose, triglycerides (TG), total cholesterol (TC), and HDL-cholesterol (HDL-C) were determined by colorimetric methods using the Cobas Integra 400 Plus (ROCHE Diagnostics, Indianapolis, IN, USA). The fasting blood insulin was measured using chemiluminescence immunoassay (Immulite 2000, Diagnostic Products Corporation, 
Los Angeles, CA). The ratio of TC to HDL-C was calculated as an index of atherogenic lipid profile. All assays were performed in duplicate according to the manufacturers' instructions.

\section{Data management}

The homeostatic model assessment (HOMA-IR), calculated as the product of basal glucose $(\mathrm{mmol} / \mathrm{L})$ and insulin $(\mu 1 \mathrm{U} / \mathrm{mL})$ levels divided by 22.5 , was used as a proxy measure of insulin resistance [31]. A continuous score representing a composite cardiometabolic risk score was derived by summing the standardized values $[\mathrm{Z}$-score $=$ (participant's value - mean value of the sample) / standard deviation)] by age and sex, of triglycerides, systolic blood pressure, ratio total cholesterol/HDL-cholesterol, HOMAIR and waist circumference as already proposed for adolescents [4,32]. Participants above 1 standard deviation (SD) of this score were classified has having a high cardiometabolic risk.

\section{Statistical Analysis}

Descriptive data are presented as means and standard deviation. Independent Two-tailed $t$-Tests for continuous variables and Chi-square for categorical variables, respectively, were used to examine sex differences. Since no significant interaction was observed between sexes (e.g., sex x muscular fitness score), all the statistical analysis was performed with both sexes together, to increase statistical power.

Partial correlations were performed to examine the associations between 251 SEADiet, cardiovascular risk factors and muscular fitness measures adjusted for sex, age and pubertal stage. The SEADiet index correlations were additionally adjusted for total energy intake. 

muscular fitness score and cardiovascular risk score, adjusted for age, sex, pubertal stage, socioeconomic status, SEADiet and cardiorespiratory fitness. Unstandardized regression coefficients were used to express the B coefficients in the regression analyses.

Analysis of covariance (ANCOVA) with Bonferroni post-hoc multiple comparison tests were used to assess the differences of cardiometabolic risk score across groups with different levels of muscular fitness score by adherence to SEADiet. For this analysis, adolescents were categorized as having a low or high adherence to SEADiet and as having a low, medium and high muscular fitness score. Covariates included were age, sex, pubertal stage, socioeconomic status, total energy intake and cardiorespiratory fitness. Furthermore, we adjusted by under-reporting energy intake, which was estimated using the ratio between reported energy intake and predicted basal metabolic rate $(31,32)$. The thresholds that defined low-energy reporters (underreporters) were 1.70 and 1.71 for girls and boys between 15 and 17 years old, and 1.67 and 1.81 for girls and boys aged 18. 'Low-energy reporter' (a categorical variable) was included in the model as a confounding. Finally, binary logistic regressions were performed to assess odds ratios (OR) and 95\% CI predicting high cardiometabolic risk according to the combined groups of muscular fitness and adherence to SEADiet; covariates included age, sex, pubertal stage, socioeconomic status, total energy intake, low-energy reporter and cardiorespiratory fitness.

A $P$ value less than 0.05 was regarded as significant. Data analysis was performed using the Statistical Package for the Social Sciences for Windows (Version 

for all). Girls presented lower values of WC, TC/HDL-C, systolic blood pressure and higher TC and HDL-C than boys $(p<0.01)$.

287 \#INSERT TABLE 1\#

Table 2 shows partial correlations for muscular fitness variables, SEADiet index and individuals cardiovascular risk factors. Muscular fitness score was negatively associated with all metabolic risk factors, and positively associated with SEADiet index. When we analysed the individual measures of muscular fitness, Curl Up was positively correlated with SEADiet and negatively correlated with all single metabolic risk factors measures and cardiovascular risk score. Push Up was also positively correlated with SEADiet, and negatively correlated with systolic blood pressure, HOMA-IR, WC and cardiovascular risk score. SEADiet index was negatively associated with systolic blood pressure, WC and cardiovascular risk score ( $\mathrm{p}<0.05$ for all).

300 \#INSERT TABLE 2\#

301 
317 \#INSERT FIGURE 1\#

318

319

\section{INSERT TABLE 3\#}

(1)

0

age, sex, pubertal stage, socioeconomic status, SEADiet index, total energy intake, lowenergy reporter and cardiorespiratory fitness (Table 3).

ANCOVA showed a significant difference between the Low muscular fitness/Low adherence to SEADiet group and the High muscular fitness/Low adherence to SEADiet and High muscular fitness/High adherence to SEADiet groups $(\mathrm{p}<0.05)$, after adjustments for age, sex, pubertal stage, socioeconomic status, total energy intake, low-energy reporter and cardiorespiratory fitness showed. We also found significant differences between Low muscular fitness/High adherence to SEADiet group and High muscular fitness/High adherence to SEADiet $\left(\mathrm{F}_{(5,452)}=5.074 p<0.001\right)$ (Figure 1).

muscular fitness/Low SEADiet had the highest odds of having a high cardiometabolic risk (OR=4.5; 95\% CI: $2.1-14 \mathrm{p}=0.001)$ followed by the High SEADiet/Low muscular fitness group (OR=3.3; 95\% CI: 1.5-11.2 p=0.046) when compared to those with High muscular fitness/High SEADiet, after adjustments for potential confounders.

Table 4. Binary logistic regression analysis showed that participants with a Low 

and cardiorespiratery fitness-on cardiovascular risk. Our results showed that muscular

fitness was negatively associated with cardiovascular risk score even after considering potential confounders such as age, sex, pubertal stage, socioeconomic status, SEADiet, total energy intake and cardiorespiratory fitness. We also found significant differences on the cardiometabolic risk score per the different levels of muscular fitness and SEADiet groups. In addition, adolescents classified with low muscular fitness and low adherence to a SEADiet had the poorest cardiometabolic profile, which also highlights the importance of the combined effect of a healthy dietary intake and high muscle strength. Participants with low muscular fitness/low SEADiet had the highest odds of having high cardiometabolic risk, followed by the high SEADiet/low muscular fitness group, when compared to those with high muscular fitness/high SEADiet, after adjustments for potential confounders

The relationship between metabolic risk factors and muscular fitness has been explored in adolescents. Previous research has reported inverse associations between muscular fitness and individual cardiovascular risk factors [33], inflammatory biomarkers [34], and clustered cardiovascular risk factors [12,13,35,36]. Recently, a 20 years follow-up study with 737 subjects aged 9,12 , or 15 years at baseline showed that childhood muscular fitness predicted adult metabolic syndrome outcomes independently of childhood cardiorespiratory fitness [37]. In addition, a recent systematic review and meta-analysis showed strong evidence for an inverse association between muscular fitness and cardiometabolic risk factors in adolescents [9]. However, none of these studies adjusted their analyses for dietary patterns, nor considered the independent and 
combined effect of muscular fitness and dietary patterns on the cardiometabolic risk in adolescents. Therefore, to the best of our knowledge, this is the first study assessing the combined associations of muscular fitness and adherence to a healthy dietary pattern, namely the SEADiet, on the cardiometabolic profile in adolescents.

Our study showed that SEADiet was inversely correlated with systolic blood pressure, WC, and a cluster of cardiometabolic risk factors. Recent studies have investigated the benefits of adherence to a SEADiet. Oliveira et al. [15] showed that higher adherence to the SEADiet was associated with a lower risk of non-fatal acute myocardial infarction. Conversely, another study with 10,231 individuals representative of the population aged 18 years and older in Spain - showed that the SEADiet was associated with a lower concentration of inflammatory markers and with reduced triglycerides, insulin, insulin resistance, and systolic blood pressure [16]. Cardiorespiratory fitness, a powerful marker of current and future health status in children and adolescents $[8,38]$, has been positively associated with adherence to the SEADiet and inversely associated with cardiovascular risk factors $[17,18]$. Importantly for this discussion, in the present investigation we considered cardiorespiratory fitness, adherence to SEADiet, and socio-economic status as confounders in our analysis between muscular fitness and cardiometabolic risk score, and the results remained significant. In addition, in the present study we matched the muscular fitness status to the SEADiet groups, which allowed us to compare differences in the cardiometabolic profile of the two groups of SEADiet (low and high adherence) by levels of muscular fitness levels. We observed that adolescents with higher muscular fitness levels exhibit the lowest cardiovascular risk, regardless of their adherence to SEADiet. These results seem to suggest that muscular fitness may play a key role in the cardiometabolic profile of adolescents, independent of several confounders. Although a cross-sectional design 
was used, our data seem to suggest that having high muscular fitness levels may somewhat overcome the deleterious effects of low adherence to healthy dietary patterns. Diet and physical activity have different effects on body composition, with both contributing to weight control and loss. However, the skeletal muscle is the major site of glucose uptake [39], a highly energetic tissue that contributes substantially to basal metabolic rate [40]. Exercise training is the most potent stimulus to increase skeletal muscle GLUT4 expression, which facilitates insulin action and glucose disposal; and, consequently, insulin resistance prevention [39]. Recently, a study comparing the effects of aerobic, strength, and combined aerobic and strength training on metabolic disorders induced by a fructose-rich diet in animal models showed that hyperinsulinemia, glucose intolerance, and insulin resistance were attenuated by aerobic physical exercise, while strength training was able to restore all these parameters to control levels. [41] In this line of thought, high muscular fitness levels are associated with better skeletal muscle function, which directly affects the metabolic function and may explain the better cardiometabolic profile in our participants. Nevertheless, the prevention of cardiometabolic risk via healthy dietary patterns remains important, although future work should also focus on promoting high muscular fitness levels.

Some limitations of our study should be taken into consideration. First, we were unable to draw cause-effect conclusions because of the cross-sectional nature of our data. Second, we cannot rule out some reporting bias because we used self-reported dietary intake data. However, the FFQ was previously tested [42] and the analyses were controlled to prevent the misreporting of energy intake.

Strengths of this study include the novelty of the analyses of combined associations of muscular fitness with adherence to a healthy dietary pattern on the cardiometabolic risk of adolescents; moreover, most studies that reported the 
relationship between physical fitness and cardiovascular risk score were confined to cardiorespiratory fitness. To our knowledge, few studies have been conducted among adolescents assessing other health-related fitness components associated with cardiometabolic risk factors. Furthermore, the use of a valid field test for muscular fitness assessment, which can be administered in school settings and allow a large number of participants to be tested simultaneously, is another strength. Indeed, this is a valuable tool for routinely measuring physical fitness in youth.

In conclusion, although our study used a cross-sectional design, our findings suggest that muscular fitness was inversely associated with a cluster of cardiometabolic risk in adolescents, independent of SEADiet adherence. Furthermore, adolescents with high levels of muscular fitness presented lower odds of having a high cardiometabolic profile, independent of their adherence to a SEADiet. Our study also suggests that the combination of poor dietary habits and low muscular fitness levels is associated with a poor cardiometabolic profile. From a public health perspective, our data seem to suggest that improving muscular fitness and adhering to a healthy diet could be an important strategy to reduce clustered cardiometabolic risk in youth. Therefore, the study highlights the importance of developing muscular fitness at these ages and seems to corroborate the international World Health Organization's recommendations of strengthening muscle and bone at least 3 times per week.

\section{ACKNOWLEDGEMENTS}

The author C.A.A.S was given Doctoral scholarship from Brazilian government by CAPES (Coordination of Improvement of Higher Education Personnel) (Proc: 9588- 
431 supported by UID/DTP/00617/2013 (FCT). The author R.S. has a Discovery Early

432 Career Research Award from the Australian Research Council (DE150101921). The 433 authors R.R.V. and J.E.C.B. are supported by Instituto Colombiano para el Desarrollo 434 de la Ciencia y la Tecnología "Francisco José de Caldas" COLCIENCIAS (Contract No 435 671-2014 Code 122265743978).

436 The authors gratefully acknowledged the participation of all adolescents and 437 their parents, teachers and schools of the Azorean Physical Activity and Health Study II. 438 They also acknowledge the cooperation of volunteer's subjects and the Research Centre 439 in Physical Activity, Health and Leisure (University of Porto) for the sponsoring the 440 LabMed Study.

441 The authors have no conflicts of interest relevant to this article to disclose. 
[1] Gersh BJ, Sliwa K, Mayosi BM, Yusuf S. Novel therapeutic concepts: The epidemic of cardiovascular disease in the developing world: Global implications.

[2] McGill H.C. J, McMahan C a., Herderick EE, Malcom GT, Tracy RE, Jack P. Origin of atherosclerosis in childhood and adolescence. Am J Clin Nutr 2000;72:1307S-1315S.

[3] Alberti SG, Zimmet P. The IDF Consensus definition of the Metablic Syndrome in Children and Adolescents. Int Diabetes Fed 2007:24. doi:2-930229-49-7.

[4] Andersen LB, Lauersen JB, Brønd JC, Anderssen SA, Sardinha LB, Steenejohannessen J, et al. A New Approach to Define and Diagnose Cardiometabolic Disorder in Children 2015;2015.

[5] Balagopal PB, De Ferranti SD, Cook S, Daniels SR, Gidding SS, Hayman LL, et al. Nontraditional risk factors and biomarkers for cardiovascular disease: mechanistic, research, and clinical considerations for youth: a scientific statement from the American Heart Association. Circulation 2011;123:2749-69. doi:10.1161/CIR.0b013e31821c7c64.

[6] Thomas NE, Williams DRR. Inflammatory factors, physical activity, and physical fitness in young people: Review. Scand J Med Sci Sport 2008;18:54356.

[7] Hansson GK. Mechanisms of disease: Inflammation, atherosclerosis, and coronary artery disease. N Engl J Med 2005;352:1685-1695+1626.

[8] Ortega FB, Ruiz JR, Castillo MJ, Sjöström M. Physical fitness in childhood and adolescence: a powerful marker of health. Int J Obes (Lond) 2008;32:1-11. doi:10.1038/sj.ijo.0803774.

[9] Smith JJ, Eather N, Morgan PJ, Plotnikoff RC, Faigenbaum AD, Lubans DR. The health benefits of muscular fitness for children and adolescents: a systematic review and meta-analysis. Sport Med 2014;44:1209-23. doi:10.1007/s40279014-0196-4.

[10] Ortega FB, Silventoinen K, Tynelius P, Rasmussen F. Muscular strength in male adolescents and premature death : cohort study of one million participants. BMJ 2012;7279:345. doi:10.1136/bmj.e7279.

[11] Ramírez-vélez R, Tordecilla-sanders A, Correa-bautista JE, Peterson MD, Garcia-hermoso A. Handgrip Strength and Ideal Cardiovascular Health among Colombian Children and Adolescents. J Pediatr 2016. doi:10.1016/j.jpeds.2016.08.099.

[12] Mota J, Vale S, Martins C, Gaya A, Moreira C, Santos R, et al. Influence of muscle fitness test performance on metabolic risk factors among adolescent girls. Diabetol Metab Syndr 2010;2:42. doi:10.1186/1758-5996-2-42.

[13] Artero EG, Ruiz JR, Ortega FB, España-Romero V, Vicente-Rodríguez G, Molnar D, et al. Muscular and cardiorespiratory fitness are independently 
associated with metabolic risk in adolescents: The HELENA study. Pediatr Diabetes 2011;12:704-12. doi:10.1111/j.1399-5448.2011.00769.x.

[14] Agostinis-Sobrinho C, Moreira C, Abreu S, Lopes L, Sardinha LB, OliveiraSantos J, et al. Muscular fitness and metabolic and inflammatory biomarkers in adolescents: Results from LabMed Physical Activity Study. Scand J Med Sci Sports 2016:1-8. doi:10.1111/sms.12805.

[15] Oliveira A, Lopes C, Rodríguez-Artalejo F. Adherence to the Southern European Atlantic Diet and occurrence of nonfatal acute myocardial infarction. Am J Clin Nutr 2010;92:211-7. doi:10.3945/ajcn.2009.29075.

[16] Guallar-Castillón P, Oliveira A, Lopes C, López-García E, Rodríguez-Artalejo F. The Southern European Atlantic Diet is associated with lower concentrations of markers of coronary risk. Atherosclerosis 2013;226:502-9. doi:10.1016/j.atherosclerosis.2012.11.035.

[17] Moreira C, Santos R, Moreira P, Lobelo F, Ruiz JR, Vale S, et al. Cardiorespiratory fitness is negatively associated with metabolic risk factors independently of the adherence to a healthydietary pattern. Nutr Metab Cardiovasc Dis 2013;23:670-6. doi:10.1016/j.numecd.2012.01.011.

[18] Liao W, Xiao D, Huang Y, Yu H, Yuan S, Chen T, et al. Combined Association of Diet and Cardiorespiratory Fitness with Metabolic Syndrome in Chinese Schoolchildren. Matern Child Health J 2016;20:1904-10. doi:10.1007/s10995016-2001-5.

[19] Peake JM, Della Gatta P, Suzuki K, Nieman DC. Cytokine expression and secretion by skeletal muscle cells: regulatory mechanisms and exercise effects. Exerc Immunol Rev 2015;21:8-25.

[20] Moreira C, Santos R, Vale S, Soares-Miranda L, Marques AI, Santos PC, et al. Metabolic syndrome and physical fitness in a sample of Azorean adolescents. Metab Syndr Relat Disord 2010;8:443-9. doi:10.1089/met.2010.0022.

[21] Welk GJ, Meredith MD. Fitnessgram/Activitygram reference guide. Dallas, TX Cooper Inst 2008;3.

[22] Lopes CM. Reprodutibilidade e Validação de um questionário semi-quantitativo de frequência alimentar. In: Alimentação e enfarte agudo do miocárdio: um estudo caso-controlo de base populacional. Tese de Doutoramento. University of Porto, 2000.

[23] Lopes C, Aro A, Azevedo A, Ramos E, Barros H. Intake and adipose tissue composition of fatty acids and risk of myocardial infarction in a male \{Portuguese\} community sample. J Am Diet Assoc J Am Diet Assoc 2007;107:276-86.

[24] Silva D, Rego C, Guerra A, Sinde S, Zara L, Moreira P. Characterization of food habits and comparative study between two methods of food assessment in adolescents. Rev Aliment Humana 2004;10:33-40.

[25] Ramos E. Health determinants in Porto adolescents- PhD Thesis. The Epiteen cohort: Porto: University of Porto;, 2006. 
[26] Leger LA, Mercier D, Gadoury C, Lambert J. The multistage 20 metre shuttle run test for aerobic fitness. J Sport Sci 1988;6:93-101. doi:10.1080/02640418808729800.

[27] Lohman TG, Roche AF, Martorell F, Martorell R. Anthropometric standardization reference manual. Champaign IL: Human Kinetics Book; 1991.

[28] Tanner JM, Whitehouse RH. Clinical longitudinal standards for height, weight, height velocity, weight velocity, and stages of puberty. Arch Dis Child 1976;51:170-9. doi:10.1136/adc.51.3.170.

[29] Mota J, Silva G. Adolescent's Physical Activity: Association with SocioEconomic Status and Parental Participation Among a Portuguese Sample. Sport Educ Soc 1999;4:193-9. doi:10.1080/1357332990040206.

[30] McCrindle BW. Assessment and management of hypertension in children and adolescents. Nat Rev Cardiol 2010;7:155-63. doi:10.1038/nrcardio.2009.231.

[31] Matthews D, Hosker J, Rudenski A, Naylor B, Treacher D, Turner R. Homeostasis Model Assessment: insulin resistance and beta-cell function from fasting plasma glucose and insulin concentrations in man. Diabetol 1985;28:4129.

[32] Bugge A, El-Naaman B, McMurray RG, Froberg K, Nielsen CH, Müller K, et al. Inflammatory markers and clustered cardiovascular disease risk factors in danish adolescents. Horm Res Paediatr 2012;78:288-96.

[33] Agostinis-Sobrinho C, Santos R, Moreira C, Abreu S, Lopes L, Oliveira-Santos J, et al. Association between serum adiponectin levels and muscular fitness in Portuguese adolescents: LabMed Physical Activity Study. Nutr Metab Cardiovasc Dis 2016;6:517-24. doi:10.1016/j.numecd.2016.02.011.

[34] Artero EG, España-Romero V, Jiménez-Pavón D, Martinez-Gómez D, Warnberg J, Gómez-Martínez S, et al. Muscular fitness, fatness and inflammatory biomarkers in adolescents. Pediatr Obes 2013;9:391-400.

[35] Steene-Johannessen J, Anderssen SA, Kolle E, Andersen LB. Low muscle fitness is associated with metabolic risk in youth. Med Sci Sports Exerc 2009;41:13617. doi:10.1249/MSS.0b013e31819aaae5.

[36] Segura-Jiménez V, Parrilla-Moreno F, Fernández-Santos JR, Esteban-Cornejo I, Gómez-Martínez S, Martinez-Gomez D, et al. Physical fitness as a mediator between objectively measured physical activity and clustered metabolic syndrome in children and adolescents: The UP\&DOWN study. Nutr Metab Cardiovasc Dis 2016:1-9. doi:10.1016/j.numecd.2016.07.001.

[37] Fraser BJ, Huynh QL, Schmidt MD, Dwyer T, Venn AJ, Magnussen CG. Childhood Muscular Fitness Phenotypes and Adult Metabolic Syndrome. Med Sci Sports Exerc 2016;48:1715-22. doi:10.1249/MSS.0000000000000955.

[38] Ruiz JR, Castro-Piñero J, Artero EG, Ortega FB, Sjöström M, Suni J, et al. Predictive validity of health-related fitness in youth: a systematic review. $\mathrm{Br} \mathbf{J}$ Sports Med 2009;43:909-23. doi:10.1136/bjsm.2008.056499. 
572

573

574

575

576

577

578

579

580

581

582

583

585

586

587

588

589

590

\section{9}

\section{4}

[39] Richter EA, Hargreaves M. Exercise, GLUT4, and skeletal muscle glucose uptake. Physiol Rev 2013;93:993-1017. doi:10.1152/physrev.00038.2012.

[40] Volaklis K a., Halle M, Meisinger C. Muscular strength as a strong predictor of mortality: A narrative review. Eur J Intern Med 2015;26:303-10. doi:10.1016/j.ejim.2015.04.013.

[41] Botezelli JD, Andressa C, Ghezzi AC, Cambri LT, Moura LP, Scariot PPM, et al. Strength Training Prevents Hyperinsulinemia , Insulin Resistance, and Inflammation Independent of Weight Loss in Fructose-Fed Animals. Sci Rep 2016;6:1-13. doi:10.1038/srep31106.

[42] Mota J, Esculcas C. Leisure-time physical activity behavior: structured and unstructured choices according to sex, age, and level of physical activity. Int J Behav Med 2002;9:111-21. doi:10.1207/S15327558IJBM0902_03.

80

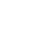

Figure 1: Means values of cardiovascular risk score stratified in tertiles of muscular fitness (low, medium, and high) in Low (low $<=4$ points) and High ( $>=5$ points) adherence to SEADiet. Adjusted for age, sex, pubertal stage, socioeconomic status, total energy intake, low-energy reporter and cardiorespiratory fitness.

* Significantly different $(\mathrm{p}<0.05)$ 


\section{Highlights}

1- Muscular fitness and adherence to the SEADiet are associated with a cardiometabolic risk score.

2- High muscular fitness levels may somewhat overcome the deleterious effects of low adherence to the SEADiet.

3- Adolescents with low muscular fitness and low adherence to the SEADiet had the poorest cardiovascular profile. 
Table 1. Participants' characteristics

\begin{tabular}{|c|c|c|c|}
\hline Characteristics & $\frac{\text { Girls }}{(n=625)}$ & $\begin{array}{c}\text { Boys } \\
(\mathrm{n}=834)\end{array}$ & $\frac{\text { Total }}{(\mathrm{n}=1462)}$ \\
\hline Age (year) & $13.5( \pm 2.1)$ & $13.6( \pm 2.1)$ & $13.5( \pm 2.1)$ \\
\hline Weight (Kg) & $48.9( \pm 10.9)$ & $51.3( \pm 13.9)$ & $50.1( \pm 12.4)$ \\
\hline Height $(\mathrm{cm})$ & $154( \pm 8)$ & $160( \pm 12)$ & $157( \pm 11)$ \\
\hline Body mass index & $20.6( \pm 3.5)$ & $20.2( \pm 3.3)$ & $20.5( \pm 3.5)$ \\
\hline $\begin{array}{l}\text { Pubic hair development: } \\
\leq \mathrm{III} / \mathrm{IV} / \mathrm{V}(\%)^{*}\end{array}$ & $55.4 / 34.3 / 10.3$ & $53.2 / 37.3 / 9.5$ & $54.3 / 35.8 / 9.9$ \\
\hline $\begin{array}{l}\text { Breast/genital development: } \\
\leq \mathrm{III} / \mathrm{IV} / \mathrm{V}(\%)^{*}\end{array}$ & $57.1 / 37.3 / 5.6$ & $59.7 / 31.1 / 9.2$ & $58.3 / 34.4 / 7.3$ \\
\hline C-Reactive Protein (mgL) & $0.96( \pm 1.6)$ & $1.18( \pm 2.0)^{*}$ & $1.04( \pm 1.8)$ \\
\hline Cardiorespiratory fitness $\mathrm{VO}_{2} \max (\mathrm{mL} / \mathrm{kg} / \mathrm{min})$ & $38.4( \pm 4.4)$ & $43.6( \pm 5.9)^{*}$ & $40.8( \pm 5.7)$ \\
\hline Standing Long Jump & $128( \pm 25)$ & $156( \pm 33)^{*}$ & $141.7( \pm 32)$ \\
\hline Handgrip (kg) & $21.1( \pm 4.9)$ & $27.1( \pm 9.9)^{*}$ & $23( \pm 8.2)$ \\
\hline \multicolumn{4}{|l|}{ Adherence to a MedDiet } \\
\hline Optimal Adherence (\%) & 20.8 & 22.7 & 21.7 \\
\hline
\end{tabular}


Table 2. Odds ratio of High Inflammatory Profile by Adherence to a Mediterranean Diet, Cardiorespiratory Fitness and Muscular Fitness.

\begin{tabular}{clcccc}
\hline \multirow{2}{*}{ Parameter } & \multicolumn{4}{c}{ High Inflammatory Profile } \\
\cline { 3 - 6 } & HighMF/HighCRF & $\begin{array}{c}\text { OR unadjusted } \\
(95 \% \text { CI })\end{array}$ & $p$-value & $\begin{array}{c}\text { OR adjusted } \\
(95 \% \text { CI })\end{array}$ & $p$-value \\
\hline \multirow{2}{*}{ Optimal } & HighMF/LowCRF & $2.1(0.9-5.2)$ & 0.331 & $1.38(0.6-3.0)$ & 0.495 \\
Adherence & LowMF/HighCRF & $1.71(0.7-4.3)$ & 0.258 & $1.23(0.5-3.1)$ & 0.667 \\
& LowMF/LowCRF & $4.1(2.5-9.0)$ & $<0.001$ & $2.3(1.2-5.0)$ & 0.045 \\
\hline \multirow{2}{*}{ Low } & HighMF/HighCRF & $1.4(0.9-2.0)$ & 0.167 & $1.27(0.8-2.1)$ & 0.342 \\
adherence & HighMF/LowCRF & $2.1(1.3-3.5)$ & 0.004 & $2.3(1.2-4.0)$ & 0.095 \\
& LowMF/HighCRF & $1.50(0.8-2.8)$ & 0.175 & $1.12(0.7-2.1)$ & 0.718 \\
& LowMF/LowCRF & $3.75(2.20-6.3)$ & $<0.001$ & $2.3(1.2-4.0)$ & 0.007 \\
\hline
\end{tabular}

OR, odds ratios; CI, confidence intervals; 1, reference category. *Adjusted for age, sex, pubertal stage, country and BMI z-score. MF; Muscular Fitness. CRF; cardiorespiratory fitness 
Table 3. Unstandardized regression coefficients examining the association of cardiovascular risk score and muscular fitness

\begin{tabular}{lcccc}
\hline \multirow{2}{*}{$\begin{array}{c}\text { Muscular Fitness } \\
\text { (score) }\end{array}$} & \multicolumn{4}{c}{ Cardiovascular risk score } \\
\cline { 2 - 5 } Model 1 & $\mathrm{B}$ & $(95 \%$ C.I) & $P$-value & $\mathrm{R}^{2}$ \\
\cline { 2 - 5 } Model 2 & -0.570 & $(-0.734:-0.406)$ & $<0.001$ & 0.092 \\
Model 3 & -0.608 & $(-0.809:-0.462)$ & $<0.001$ & 0.119 \\
Model 4 & -0.621 & $(-0.796:-0.446)$ & $<0.001$ & 0.121 \\
\hline
\end{tabular}

B: Unstandardized coefficients;

Model 1- Unadjusted model.

Model 2 - Adjusted, age, sex, pubertal stage and socioeconomic status.

Model 3 - Model 2 plus adjustment for SEADiet index.

Model 4 - Model 3 plus adjustment for cardiorespiratory fitness and total energy intake and low-energy reporter (Fully adjusted model) 
Table 4. Odds ratio of high cardiometabolic risk by muscular fitness levels and Adherence to SEADiet

\begin{tabular}{lccc}
\hline \multirow{2}{*}{ Parameter } & \multicolumn{2}{c}{ High cardiometabolic risk } \\
\cline { 2 - 4 } & High MF & OR adjusted ${ }^{*}(95 \% \mathrm{C} . \mathrm{I})$ & $P$-value \\
\hline \multirow{3}{*}{ High SEADiet } & 1 & 1 \\
& Medium MF & $1.83(0.5-6.2)$ & 0.334 \\
& Low_MF & $3.30(1.5-11.2)$ & 0.046 \\
\multirow{2}{*}{ Low SEADiet } & High MF & $0.8(0.17-4.1)$ & 0.830 \\
& Medium MF & $2.87(0.80-9.0)$ & 0.100 \\
& Low MF & $4.5(2.1-14.0)$ & 0.001 \\
\hline
\end{tabular}

OR, odds ratios; CI, confidence intervals; 1 , reference category. MF, muscular fitness

*Adjusted for age, sex, pubertal stage, socioeconomic status, total energy intake, low-energy reporter and cardiorespiratory fitness. 
